\title{
Autism is Physiological, Nonautism is Pathological Biologically Speaking
}

\author{
Otto E. Rossler*
}

Faculty of Science, University of Tuebingen, Auf der Morgenstelle 8, 72076 Tuebingen, Germany

${ }^{\star}$ Corresponding author: Otto E. Rossler, Faculty of Science, University of Tuebingen, Auf der Morgenstelle 8, 72076 Tuebingen, Germany; Phone: +497071290

Received: July 09, 2020; Accepted: July 23, 2020; Published: July 31, 2020

\begin{abstract}
A brief functional and historical analysis is offered of the question, what is the physiological difference between human beings and other animals? All animals are autistic, even those equipped with a much higher intelligence than the human one is, like whales, for example. So the non-autism of most human beings is what needs explanation.
\end{abstract}

Nonautism is the consequence of a misunderstanding - a creative misunderstanding, that is. It consists in the suddenly conceived suspicion that the other was trying to make you happy - an absurd idea. While it is true that brood-caring animals get rewarded by signs of being satisfied emitted by the young, as a confirmation of their own brood-rearing efforts being momentarily successful, this reward is purely one-directional. There is no reason conceivable why the offspring ought to be rewarded by the adults being successful in anything (like feeding them). It is always only the effect that counts in biology. Survival is the currency, nothing else.

But in humans, the young are being rewarded by the displayed joy of adults? Exactly this is the case. Humans are a pathological species in this respect. Actually, such a functional "wiring" can be proved to be pathological in evolution - that is, to be subject to predictable fast evolutionary elimination, should it have arisen once. How, then, could this pathological type of wiring actually arise in evolution, as it did with the human species? The answer is: By accident. It is a very specific accident at stake here that can be pinpointed.

It is the phenomenological overlap between the innate (wired-in) expressions of bonding on the one hand and the expression of wellbeing and friskiness on the other. In many animals, this expression of friskiness and happiness and enthusiasm, if shown by the young, has a rewarding effect on the adults, for it signals that their attempts at successful brood-rearing are on the right track. It is sweet to watch this rewardability channel in action. Many predatorial animals are socially wired in this way among each other.

But this is not our question here. How come that the young are rewarded by the playful friskiness of the adults in some species? The "playing mode" has this very structure in many social animals. Play occurs when more "serious" needs are satisfied and the partners can invest energy in silly, that is not survival relevant activities, as a means to tighten their social bonds. Play makes peace and friendship. But our context is non-autism. How could it arise in one species so far? What arose here is very easy to see: Mutual rewardability by the expression of playfulness on the other side. By the playful mode. Homo ludens. All social animals are wired like this in play.

But there is a risk involved here: The suspicion of benevolence encountered arising on one side. For play is highly rewarding. Can there for this reason arise a "serious" component?. This is what has occurred in the human species. It happens in play as in the other high-performance social animals, but not only in play. "Homo ludens" (humans are players) even when not playing? The positive feedback that arises in play can indeed be maintained so as to enter all other social activities. The smile-laughter is the play mode. Wolves can enter the same mutually inspired frenzy. They are very much like human beings even though the expressions are not so much shown on the face as with the tail.

So what is the functional difference? That wolves are not mirror competent is the answer. So the "suspicion of benevolence encountered" cannot arise in the wolf. But it can and does - in the human wolf. The "werwolf" sagas show how irritated humans are by the close emotional similarity of the wolf. There are other highly social animals like dolphins and whales and also some octopuses and giant creatures from the depth. And don't forget the elephants. Are we humans not wired socially in much the same manner, even if being somewhat less intelligent? There is one difference functionally speaking, however.

What is it? The smile of happiness, the expression of happiness, is at the same time the smile of bonding. It is the same expression. But is this not the same thing with the wolf? Yes, it is. And presumably so with the African wild dog, Lycaon pictus, as well? And maybe with some sepiae yet to be investigated.

Now I have left you in the dark long enough about what we are headed for: The positive feedback of the smile acting symmetrically. Not only when bonding, but also when merely playfully happy. Or just happy. The consequence is the sudden invention of the suspicion of 
one's encountering benevolence. It is absolutely nonsensical, right? There is nothing but hard-wired couplings here. There is no room for such humanistic notions like "benevolence." But actually, this transgression is exactly what is happening and taking place here between us.

It could happen also between orcas. Or between an orca and a human. Or between Kanzi and his wonderful trainer. Not because but in spite of the silly machine inserted in between them. Big revolutionary progress sometimes takes unnecessary roundabout ways. We will be able to ask Kanzi. He will understand the question and tell us. Come on: It can't possibly be that simple! But it is. We walked together through a nice little wood of well-defined observational biological notions above and do now suddenly see ourselves in a mirror. Yes, the question posed at the outset - the onset of the suspicion of encountering benevolence - is really being raised here.

Will the reader tolerate having been pulled into this nightmare of a daydream: Of a functional understanding of what benevolence and love is? On a level infinitely more powerful than the other social domain of sex is, for example? The reader may feel that we will have to come back to this fascinating topic together again? Take care. 